\title{
Patterns of care for patients with nasopharyngeal carcinoma (KROG 11-06) in South Korea
}

\author{
Soo Yoon Sung, MD¹, Min Kyu Kang, MD², Chul Seung Kay, MD³ Ki Chang Keum, MD,

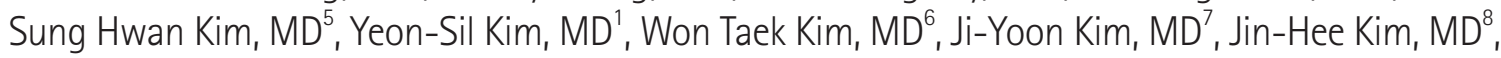 \\ Sung Ho Moon, MD ${ }^{9}$ Yong Chan Ahn, MD ${ }^{10}$, Young Taek Oh, MD ${ }^{11}$, Hong-Gyun Wu, MD ${ }^{12}$, \\ Chang-Geol Lee, MD ${ }^{4}$, Woong-Ki Chung, MD ${ }^{13}$, Kwan Ho Cho, MD ${ }^{9}$, Moon-June Cho, MD ${ }^{14}$, Jin Hwa Choi, MD ${ }^{15}$ \\ ${ }^{1}$ Department of Radiation Oncology, Seoul St. Mary's Hospital, The Catholic University of Korea College of Medicine, Seoul; \\ ${ }^{2}$ Department of Radiation Oncology, Yeungnam University College of Medicine, Daegu; \\ ${ }^{3}$ Department of Radiation Oncology, Incheon St. Mary's Hospital, The Catholic University of Korea College of Medicine, Incheon; \\ ${ }^{4}$ Department of Radiation Oncology, Yonsei Cancer Center, Yonsei University College of Medicine, Seoul; \\ ${ }^{5}$ Department of Radiation Oncology, St. Vincent's Hospital, The Catholic University of Korea College of Medicine, Suwon; \\ ${ }^{6}$ Department of Radiation Oncology, Pusan National University Hospital, Pusan National University School of Medicine, Busan; \\ ${ }^{7}$ Department of Radiation Oncology, Yeouido St. Mary's Hospital, The Catholic University of Korea College of Medicine, Seoul; \\ ${ }^{8}$ Department of Radiation Oncology, Dongsan Medical Center, Keimyung University School of Medicine, Daegu; \\ ${ }^{9}$ Department of Radiation Oncology, Proton Therapy Center, Research Institute and Hospital, National Cancer Center, Goyang; \\ ${ }^{10}$ Department of Radiation Oncology, Samsung Medical Center, Sungkyunkwan University School of Medicine, Seoul; \\ "Department of Radiation Oncology, Ajou University School of Medicine, Suwon; \\ ${ }^{12}$ Department of Radiation Oncology, Seoul National University College of Medicine, Seoul; \\ ${ }^{13}$ Department of Radiation Oncology, Chonnam National University Medical School, Gwangju; \\ ${ }^{14}$ Department of Radiation Oncology, Chungnam National University School of Medicine, Daejeon; \\ ${ }^{15}$ Department of Radiation Oncology, Chung-Ang University Hospital, Seoul, Korea
}

Purpose: To investigate the patterns of care for patients with nasopharyngeal carcinoma (NPC) in South Korea.

Materials and Methods: A multi-institutional retrospective study was performed (Korean Radiation Oncology Group [KROG] 11-06) on a total of 1,445 patients from 15 institutions.

Results: Of the 1,445 patients, more than half were stages III (39.9\%) and IV (35.8\%). In addition to patterns of care, we also investigated trends over time with the periods 1988-1993, 1994-2002, and 2003-2011. The frequencies of magnetic resonance imaging and positron emission tomography-computed tomography were markedly increased in the third period compared to previous 2 periods. Concurrent chemoradiation (CCRT) was performed on 894 patients (61.9\%), neoadjuvant chemotherapy on 468 patients (32.4\%), and adjuvant chemotherapy on 366 patients (25.3\%). Of stage II-IV patients, CCRT performed on 78.8\% in 20032011 compared to $15.0 \%$ in 1988-1993. For patients treated with CCRT, cisplatin was the most commonly used agent in $81.3 \%$ of patients. Over the periods of time, commonly used radiotherapy (RT) techniques were changed from 2-dimensional RT (1988-1993, 92.5\%) to 3-dimensional RT (2003-2011, 35.5\%) or intensity-modulated RT (IMRT; 2003-2011, 56.5\%). Median RT doses given to primary tumors, high-risk lymphatics, and low-risk lymphatics were $70.0 \mathrm{~Gy}, 58.1 \mathrm{~Gy}$, and $48.0 \mathrm{~Gy}$, respectively. Adoption of IMRT increased the dose per fraction and escalated total radiation dose.

Conclusion: Assessment of the patterns of care for NPC patients in South Korea demonstrated that management for NPC including diagnostic imaging, treatment regimen, RT techniques and dose schedule, advanced in accordance with the international guidelines.

Keywords: Nasopharyngeal neoplasms, Patterns of care, Radiotherapy, Korea

Received 21 May 2015, Revised 17 June 2015, Accepted 7 August 2015.

Correspondence: Yeon-Sil Kim, MD, Department of Radiation Oncology, Seoul St. Mary's Hospital, The Catholic University of Korea College of Medicine, 222 Banpo-daero, Seocho-gu, Seoul 06591, Korea. Tel: +82-2-2258-1501, Fax: +82-2-22581532, E-mail: yeonkim7@catholic.ac.kr

(c) This is an Open Access article distributed under the terms of the Creative Commons Attribution Non-Commercial License (http://creativecommons.org/ licenses/by-nc/4.0/) which permits unrestricted non-commercial use, distribution, and reproduction in any medium, provided the original work is properly cited.

www.e-roj.org 


\section{Introduction}

Nasopharyngeal carcinoma (NPC) shows markedly geographic and racial variations in incidence. In southern China, NPC is the most common head and neck cancer, but in the Western world, it is a rare disease with an incidence of less than 1 per 100,000 [1]. South Korea is known to show an intermediate incidence, which is lower than that of the endemic area and higher than that of the Western world. Annual report of cancer statistics in South Korea presents that a total of 379 patients were diagnosed as NPC in 2012, accounting for $0.2 \%$ of all malignancies [2].

Because of difficulty in surgical resection with an adequate margin, radiotherapy (RT) is the gold standard treatment of NPC $[3,4]$. Current guidelines recommend RT alone for the treatment of patients of stage I and concurrent chemoradiotherapy (CCRT) for the treatment of those of higher stages [5]. However, adequate RT planning is challenging because critical organs including brainstem, temporal lobe and parotid glands are closely located to nasopharynx. It is still uncertain whether adjuvant or neoadjuvant chemotherapy provide an additional benefit over definitive CCRT. Variations in details of RT dose prescription or decision of adjuvant treatment might exist. Reviewing the current patterns of care in multiple institutions would be valuable to each clinician to decide treatment policy in daily clinic. Therefore, we conducted a multi-institutional study of NPC to assess current clinical practice.

\section{Materials and Methods}

To assess the patterns of care, a multi-institutional retrospective study of NPC (Korean Radiation Oncology Group [KROG] 1106) was performed through the head and neck committee of the Korean Society of Radiation Oncology. Data were collected from the medical records of 1,476 primary NPC patients treated at 15 institutions in South Korea between September 1988 and October 2011. Eligible criteria were as follows: 1) pathologically proven NPC; 2) stage I to IVb NPC according to the American Joint Committee on Cancer (AJCC) staging, the 6th edition; 3 ) age over 18 years; and 4) the Eastern Cooperative Oncology Group (ECOG) performance status $\leq 2$. Patients who had undergone previous surgery or RT to the head and neck, and those with distant metastasis at initial diagnosis were excluded from the study.

In this study, to assess the patterns of care, we collected the data of patient and tumor characteristics, diagnostic modality, RT technique, RT dose schedule, chemotherapy schedule, treatment outcomes, and salvage treatment. Cases with missing data were included in analysis to investigate as many patients as possible. In total, 31 patients were excluded. Reasons for exclusion were as follows: 7 patients below 18 years, 2 patients with ECOG 3, 16 patients given lower than $45 \mathrm{~Gy}$ to primary mass, and 6 patients with lack of data of RT dose. Finally 1,445 patients were analyzed.

Prior to patient enrollment, Institutional Review Board approval was obtained at each participating center, and data of eligible patients were collected through web-based system developed by KROG and were transferred to the KROG Data Center (National Cancer Center, Goyang, Korea). RT, chemotherapy, toxicity, recurrence, and survival reports of all patients were reviewed according to a centralized qualityassurance program.

Response was evaluated according to World Health Organization (WHO) criteria. Overall survival (OS) was defined as the time from pathologic diagnosis of NPC to death of all cause. Recurrences were analyzed to evaluate the failure pattern. Recurrences in the primary site and the cervical lymphatic area were defined as locoregional ones. Recurrences involving other sites were regarded as distant metastasis. Survival time was estimated by the Kaplan-Meier method. Survival differences over time were analyzed using the log-rank test. A p-value of $<0.05$ (two-sided test) was considered significant.

\section{Results}

\section{Patient characteristics}

Patient characteristics are listed in Table 1. The median age of the 1,445 patients was 51 years and ranged between 18 and 86 years. The number of male patients was 1,054 (72.9\%). Based on WHO classification published in 1991, histopathological groups are divided into two major types: keratinizing squamous cell carcinoma in 225 patients (15.6\%) and nonkeratinizing carcinoma in 1,176 patients (81.4\%). Epstein-Barr virus (EBV) status was available only in 258 patients, of whom $164(11.3 \%)$ were positive for EBV. When EBV status was compared according to pathologic types, patients with nonkeratinizing carcinoma showed a higher incidence of EBV than those with keratinizing squamous cell carcinoma (68.8\% vs. 25.9\%). AJCC stages were as follows: stage I, 71 patients (4.9\%); stage II, 279 patients (19.3\%); stage III, 577 patients (39.9\%); and stage IV, 518 patients (35.8\%).

\section{Diagnosis}

The most common chief complaint was a palpable neck mass 
Table 1. Patients' characteristics

\begin{tabular}{|c|c|}
\hline Characteristics & No. $(\%)$ \\
\hline Age (yr), median (range) & $51(18-86)$ \\
\hline \multicolumn{2}{|l|}{ Gender } \\
\hline Male & $1,054(72.9)$ \\
\hline Female & $391(27.1)$ \\
\hline \multicolumn{2}{|l|}{ ECOG performance status } \\
\hline $0-1$ & $1,271(88.0)$ \\
\hline 2 & $65(4.5)$ \\
\hline Unknown & $109(7.5)$ \\
\hline \multicolumn{2}{|l|}{ Pathology } \\
\hline Keratinizing SqCC & $225(15.6)$ \\
\hline Non-keratinizing carcinoma & $1,176(81.4)$ \\
\hline Others & $16(1.1)$ \\
\hline Unknown & $28(1.9)$ \\
\hline \multicolumn{2}{|l|}{ EBV } \\
\hline Positive & $164(11.3)$ \\
\hline Negative & $94(6.5)$ \\
\hline Unknown & $1,187(82.1)$ \\
\hline \multicolumn{2}{|l|}{ T stage } \\
\hline $\mathrm{T} 1$ & $404(28.0)$ \\
\hline $\mathrm{T} 2$ & $393(27.2)$ \\
\hline T3 & $282(19.5)$ \\
\hline $\mathrm{T} 4$ & $365(25.3)$ \\
\hline Unknown & $1(0.1)$ \\
\hline \multicolumn{2}{|l|}{$\mathrm{N}$ stage } \\
\hline No & 266 (18.4) \\
\hline N1 & $380(26.3)$ \\
\hline N2 & $624(43.2)$ \\
\hline N3 & $174(12.0)$ \\
\hline Unknown & $1(0.1)$ \\
\hline \multicolumn{2}{|l|}{ AJCC stage } \\
\hline I & $71(4.9)$ \\
\hline$\|$ & 279 (19.3) \\
\hline III & 577 (39.9) \\
\hline IV & $518(35.8)$ \\
\hline
\end{tabular}

ECOG, Eastern Cooperative Oncology Group; SqCC, squamous cell carcinoma; AJCC, American Joint Committee on Cancer; EBV, Epstein-Barr virus.
(41.4\%). Nasal symptoms (epistaxis, nasal stuffiness, and rhinorrhea) were observed in $25.8 \%$ of the patients. Aural symptoms (tinnitus, hearing impairment, and otorrhea) were observed in $16.5 \%$ of the patients, and cranial nerve palsy was observed in $8.9 \%$. Other complaints were headache (4.7\%), incidental finding (1.2\%), throat symptom (1.2\%), and pain (1.1\%).

Neck computed tomography (CT, 94.1\%), neck magnetic resonance imaging (MRI, 69.8\%), positron emission tomography-computed tomography (PET-CT, 46.6\%), bone scan (35.8\%), and abdominal ultrasonography (US) or CT (29.4\%) were used for diagnostic imaging. Commonly used imaging studies were changed over the periods studied. Neck CT was performed on almost all patients in the 3 periods (1988-1993, 97.5\%; 1994-2002, 92.1\%; and 2003-2011, 95.1\%). The frequency of MRI was markedly increased in the third period compared to previous 2 periods (1988-1993, 13.2\%; 1994 2002, 55.2\%; and 2003-2011, 81.7\%). PET-CT was also used more frequently in the third period compared to previous 2 periods (1988-1993, 2.8\%; 1994-2002, 2.9\%; and 2003-2011, $72.9 \%$ ). However, bone scan, abdominal $\mathrm{CT}_{\text {, and abdominal US }}$ were decreased in the third period compared to previous 2 periods-bone scan (1988-1993, 71.8\%; 1994-2002, 71.1\%; and 2003-2011, 16.6\%), abdominal CT and abdominal US (19881993, 80.6\%; 1994-2002, 55.7\%; and 2003-2011, 14.7\%). Details of diagnostic imaging studies are shown in Table 2.

\section{Treatment}

Of the 1,445 patients, 894 (61.9\%) received CCRT and 551 (38.1\%) received RT alone. Neoadjuvant chemotherapy was given before RT to 468 patients (32.4\%) and adjuvant chemotherapy was given after RT to 366 patients (25.3\%). Grouping the patients by the treatment modalities, CCRT alone without neoadjuvant or adjuvant chemotherapy was the most common treatment regimen (27.5\%). CCRT followed by adjuvant chemotherapy (21.3\%) and RT alone without

Table 2. Changes in diagnostic modalities over the periods studied

\begin{tabular}{lcccc}
\hline Imaging modality & $1988-1993$ & $1994-2002$ & $2003-2011$ & Total period \\
\hline Neck CT & $39(97.5)$ & $455(92.1)$ & $865(95.1)$ & $1,359(94.1)$ \\
Neck MRI & $5(13.2)$ & $265(55.2)$ & $738(81.7)$ & $1,008(69.8)$ \\
PET-CT & $1(2.8)$ & $14(2.9)$ & $658(72.9)$ & $673(46.6)$ \\
Bone scan & $28(71.8)$ & $344(71.1)$ & $146(16.6)$ & $518(35.8)$ \\
Abdomen CT or US & $29(80.6)$ & $267(55.7)$ & $129(14.7)$ & $425(29.4)$ \\
\hline
\end{tabular}

Values are presented as number (\%).

CT, computed tomography; MRI, magnetic resonance imaging; PET-CT, positron emission tomography-computed tomography; US, ultrasonography. 
neoadjuvant or adjuvant chemotherapy (18.5\%) were the next common treatment regimens. The more details are presented in Table 3.

Treatment regimens showed a difference between the AJCC stages. Patients with stage III and IV disease, received CCRT more often than those with stage I and II (stage I, 14.1\%; stage II, 53.4\%; stage III, 64.6\%; and stage IV, 69.9\%). Neoadjuvant and adjuvant chemotherapies were also delivered more often to patients with more advanced diseasesneoadjuvant chemotherapy (stage I, 8.5\%; stage II, 29.4\%; stage III, 35.4\%; and stage IV, 34.0\%), adjuvant chemotherapy (stage I, 0\%; stage II, 21.1\%; stage III, 26.5\%; and stage IV,

Table 3. Combination of treatment regimens

\begin{tabular}{lc}
\hline & No. (\%) \\
\hline Treatment & \\
RT & $894(61.9)$ \\
CCRT & $551(38.1)$ \\
RT alone & \\
Neoadjuvant CTx & $468(32.4)$ \\
$\quad$ Yes & $977(67.6)$ \\
$\quad$ No & \\
Adjuvant CTx & $366(25.3)$ \\
Yes & $1,079(74.7)$ \\
$\quad$ No & \\
Combination of treatment modalities & $267(18.5)$ \\
RT alone & $398(27.5)$ \\
CCRT alone & $4(0.3)$ \\
RT + adjuvant CTx & $308(21.3)$ \\
CCRT + adjuvant CTx & $251(17.4)$ \\
Neoadjuvant CTx + RT & $163(11.3)$ \\
Neoadjuvant CTx + CCRT & $29(2.0)$ \\
Neoadjuvant CTx + RT + adjuvant CTx & $25(1.7)$ \\
Neoadjuvant CTx + CCRT + adjuvant CTx &
\end{tabular}

RT, radiotherapy; CCRT, concurrent chemoradiotherapy; CTx, chemotherapy.
29.7\%). Treatment regimens also showed a difference over the periods. CCRT increased remarkably from 15.0\% in 1988-1993 to $75.1 \%$ in $2003-2011$. Neoadjuvant chemotherapy steadily declined from $55.0 \%$ to $28.5 \%$ during studied period and adjuvant chemotherapy did not show a remarkable change after 1994 (Table 4).

Of the 894 patients who received CCRT, 768 (85.9\%) completed chemotherapy (Table 5). Weekly scheduled chemotherapy was given to 350 patients (39.1\%). More

Table 5. Concurrent chemotherapy schedule and regimen

\begin{tabular}{lc}
\hline \multicolumn{1}{c}{ Variable } & No. (\%) \\
\hline Completion & $768(85.9)$ \\
Completed & $103(11.5)$ \\
Not completed & $23(2.6)$ \\
Unknown & \\
Schedule & $350(39.1)$ \\
Weekly & $66(18.9)$ \\
$1-4$ cycles & $264(75.5)$ \\
$\geq 5$ cycles & $20(5.7)$ \\
Unknown & $511(57.2)$ \\
Every 3 or 4 weeks & $24(4.7)$ \\
1 cycle & $465(91.0)$ \\
$2-3$ cycles & $20(4.0)$ \\
$\geq 4$ cycles & $2(0.4)$ \\
Unknown & $33(3.7)$ \\
Unknown & \\
Chemotherapy regimen & $727(81.3)$ \\
Cisplatin & $72(8.1)$ \\
FP & $26(2.9)$ \\
DP & $12(1.3)$ \\
FDP & $44(4.9)$ \\
Miscellaneous & $13(1.5)$ \\
Unknown & \\
\hline
\end{tabular}

FP, 5-fluorouracil + cisplatin; DP, docetaxel + cisplatin; FDP, 5-fluorouracil + docetaxel + cisplatin.

Table 4. Treatment regimens over the periods studied

\begin{tabular}{|c|c|c|c|c|}
\hline Treatment & 1988-1993 & 1994-2002 & 2003-2011 & Total period \\
\hline \multicolumn{5}{|l|}{ RT } \\
\hline CCRT & $6(15.0)$ & 205 (41.4) & $683(75.1)$ & $894(61.9)$ \\
\hline RT alone & $34(85.0)$ & $290(58.6)$ & $227(24.9)$ & $551(38.1)$ \\
\hline \multicolumn{5}{|c|}{ Neoadjuvant chemotherapy } \\
\hline Yes & $22(55.0)$ & 187 (37.8) & $259(28.5)$ & $468(32.4)$ \\
\hline No & $18(45.0)$ & $308(62.2)$ & $651(71.5)$ & $977(67.6)$ \\
\hline \multicolumn{5}{|c|}{ Adjuvant chemotherapy } \\
\hline Yes & $3(7.5)$ & $113(22.8)$ & $250(27.5)$ & $366(25.3)$ \\
\hline No & $37(92.5)$ & $382(77.2)$ & $660(72.5)$ & $1,079(74.7)$ \\
\hline
\end{tabular}

Values are presented as number (\%).

$\mathrm{RT}$, radiotherapy; CCRT, concurrent chemoradiotherapy. 
than five cycles were given to $75.5 \%$ of the 350 patients. Approximately $57.2 \%$ of the patients received chemotherapy every 3 or 4 weeks, and most patients (91.0\%) were given two or three cycles of chemotherapy. The most commonly used chemotherapy regimen in CCRT was cisplatin (81.3\%). Neoadjuvant chemotherapy and adjuvant chemotherapy were completed in $82.7 \%$ and $80.3 \%$ of patients, respectively. In both neoadjuvant and adjuvant chemotherapies, 5-fluorouracil/ cisplatin was the most commonly used regimen (neoadjuvant chemotherapy, 49.8\%; adjuvant chemotherapy, 87.2\%). More details about neoadjuvant and adjuvant chemotherapies are

Table 6. Schedule and regimen of neoadjuvant chemotherapy and adjuvant chemotherapy

\begin{tabular}{|c|c|}
\hline & No. (\%) \\
\hline \multicolumn{2}{|c|}{ Neoadjuvant chemotherapy } \\
\hline \multicolumn{2}{|c|}{ Completion } \\
\hline Completed & $387(82.7)$ \\
\hline Not completed & $21(4.5)$ \\
\hline Unknown & $60(12.8)$ \\
\hline \multicolumn{2}{|l|}{ Regimen } \\
\hline $\mathrm{FP}$ & $233(49.8)$ \\
\hline FDP & $101(21.6)$ \\
\hline DP & 65 (13.9) \\
\hline Miscellaneous & $54(11.5)$ \\
\hline Unknown & $15(3.2)$ \\
\hline \multicolumn{2}{|l|}{ Cycle } \\
\hline 1 & $39(8.3)$ \\
\hline 2 & $82(17.5)$ \\
\hline 3 & $308(65.8)$ \\
\hline$\geq 4$ & $24(5.1)$ \\
\hline Unknown & $15(3.2)$ \\
\hline \multicolumn{2}{|c|}{ Adjuvant chemotherapy } \\
\hline \multicolumn{2}{|l|}{ Completion } \\
\hline Yes & $294(80.3)$ \\
\hline No & $60(16.4)$ \\
\hline Unknown & $12(3.3)$ \\
\hline \multicolumn{2}{|l|}{ Regimen } \\
\hline $\mathrm{FP}$ & $319(87.2)$ \\
\hline FDP & $18(4.9)$ \\
\hline DP & $9(2.5)$ \\
\hline Miscellaneous & $18(4.9)$ \\
\hline Unknown & $2(0.5)$ \\
\hline \multicolumn{2}{|l|}{ Cycle } \\
\hline $1-2$ & $90(24.6)$ \\
\hline 3 & $199(54.4)$ \\
\hline 4 & $55(15.0)$ \\
\hline$\geq 5$ & $21(5.8)$ \\
\hline Unknown & $1(0.3)$ \\
\hline
\end{tabular}

FP, 5-fluorouracil + cisplatin; DP, docetaxel + cisplatin; FDP, 5-fluorouracil + docetaxel + cisplatin. shown in Table 6.

As for RT, 98.1\% of the patients completed the whole course of treatment. Patients received RT using 4 techniques: the two-dimensional RT (2DRT) technique (24.6\%), 2DRT and 3DRT combined technique (11.7\%), 3DRT technique (28.0\%), and intensity-modulated RT (IMRT) technique (35.7\%). Changes in the four RT techniques over time were observed. The 2DRT technique was performed on 92.5\% of the patients in 1988-1993. In 2003-2011, the 3DRT and IMRT techniques were on $35.5 \%$ and $56.5 \%$ of the patients, respectively (Fig. 1). Radiation target volume was grouped as primary nasopharyngeal tumor, grossly enlarged nodes, high risk lymphatic chain and low risk lymphatic chain. High risk lymphatic chain was defined as the involved cervical nodal area. Low risk lymphatic chain was defined as the clinically and radiologically uninvolved cervical nodal areas, deemed as elective nodal regions. Different RT dose was given to each target volume, respectively. For the primary tumor and gross nodes, a radiation dose of 66-72 Gy was given to the most patients (Table 7). The median dose for primary tumors was $70.0 \mathrm{~Gy}$, and that for gross nodes was $67.5 \mathrm{~Gy}$. The conventional daily dose (1.8-2.0 Gy) was used to two-thirds of the patients. A fractional dose of over $2.0 \mathrm{~Gy}$ was given to approximately $30 \%$ of the patients. The median dose given to high-risk lymphatic chain was $58.1 \mathrm{~Gy}$, and that to low-risk lymphatic chain was $48.0 \mathrm{~Gy}$. A daily dose to lymphatic chains was rarely elevated over $2.0 \mathrm{~Gy}$. When RT doses were compared according to RT techniques, the percentage of patients who received 66-72 Gy to primary tumor was not quite different

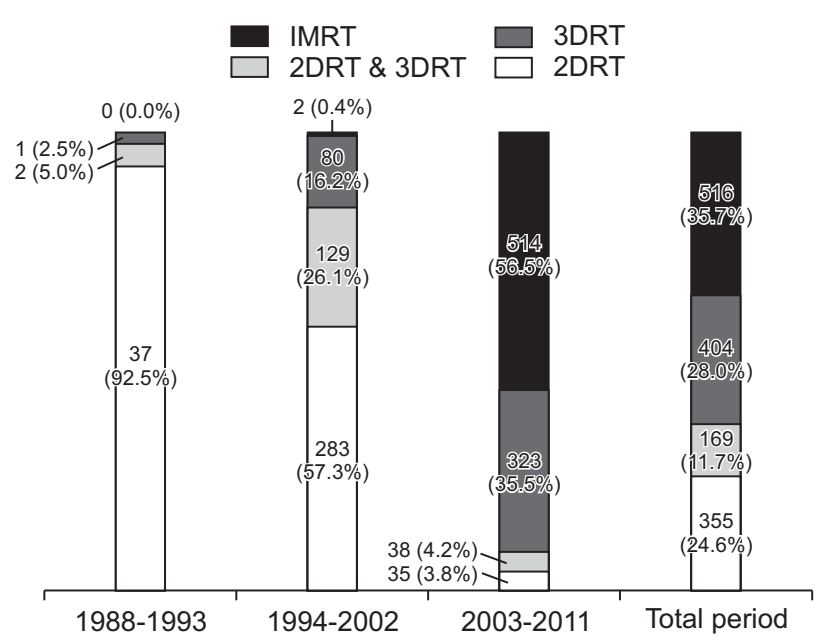

Fig. 1. Trend in radiotherapy technique over the periods studied. IMRT, intensity-modulated radiotherapy; 3DRT, three-dimensional radiotherapy; 2DRT, two-dimensional radiotherapy. 
Table 7. Radiotherapy dose schedule

\begin{tabular}{|c|c|}
\hline Dose prescription & Value \\
\hline \multicolumn{2}{|l|}{ Primary tumor dose } \\
\hline Total dose (Gy) & $70.0(45.0-100.2)$ \\
\hline$\leq 60$ & $36(2.5)$ \\
\hline$>60$ and $\leq 66$ & $245(17.0)$ \\
\hline$>66$ and $\leq 72$ & $1,030(71.3)$ \\
\hline$>72$ & 134 (9.3) \\
\hline Unknown & $0(0)$ \\
\hline Daily dose (Gy) & $2.0(1.8-3.0)$ \\
\hline$\geq 1.8$ and $\leq 2.0$ & 972 (67.3) \\
\hline$>2.0$ and $\leq 2.2$ & $174(12.0)$ \\
\hline$>2.2$ and $\leq 2.4$ & 264 (18.3) \\
\hline$>2.4$ & $3(0.2)$ \\
\hline Unknown & $32(2.2)$ \\
\hline \multicolumn{2}{|l|}{ Gross node dose } \\
\hline Total dose (Gy) & $67.5(30.6-80.0)$ \\
\hline$\leq 60$ & $237(16.4)$ \\
\hline$>60$ and $\leq 66$ & $267(18.5)$ \\
\hline$>66$ and $\leq 72$ & $554(38.3)$ \\
\hline$>72$ & $44(3.0)$ \\
\hline Unknown & $353(23.7)$ \\
\hline Daily dose (Gy) & $2.0(1.8-3.0)$ \\
\hline$\geq 1.8$ and $\leq 2.0$ & $724(50.1)$ \\
\hline$>2.0$ and $\leq 2.2$ & $127(8.8)$ \\
\hline$>2.2$ and $\leq 2.4$ & $247(17.1)$ \\
\hline$>2.4$ & $2(0.1)$ \\
\hline Unknown & $345(23.9)$ \\
\hline \multicolumn{2}{|l|}{ High risk lymphatic chain } \\
\hline Total dose (Gy) & $58.1(30.6-75.6)$ \\
\hline$\leq 50$ & $97(6.7)$ \\
\hline$>50$ and $\leq 55$ & $306(21.2)$ \\
\hline$>55$ and $\leq 60$ & $423(29.3)$ \\
\hline$>60$ & $88(6.1)$ \\
\hline Unknown & $531(36.7)$ \\
\hline Daily dose (Gy) & $1.8(1.5-2.5)$ \\
\hline$<1.8$ & $34(2.4)$ \\
\hline$\geq 1.8$ and $\leq 2.0$ & $782(54.1)$ \\
\hline$>2.0$ & $98(6.8)$ \\
\hline Unknown & $531(36.7)$ \\
\hline \multicolumn{2}{|l|}{ Low risk lymphatic chain } \\
\hline Total dose (Gy) & $48.0(5.0-71.2)$ \\
\hline$\leq 45$ & $434(30.0)$ \\
\hline$>45$ and $\leq 50$ & 192 (13.3) \\
\hline$>50$ and $\leq 55$ & $289(20.0)$ \\
\hline$>55$ & $89(6.2)$ \\
\hline Unknown & $441(30.5)$ \\
\hline Daily dose (Gy) & $1.8(1.4-2.0)$ \\
\hline$<1.6$ & $4(0.3)$ \\
\hline$\geq 1.6$ and $<1.8$ & $186(12.9)$ \\
\hline$\geq 1.8$ and $\leq 2.0$ & $814(56.3)$ \\
\hline Unknown & $441(30.5)$ \\
\hline
\end{tabular}

Values are presented as median (range) or number (\%).

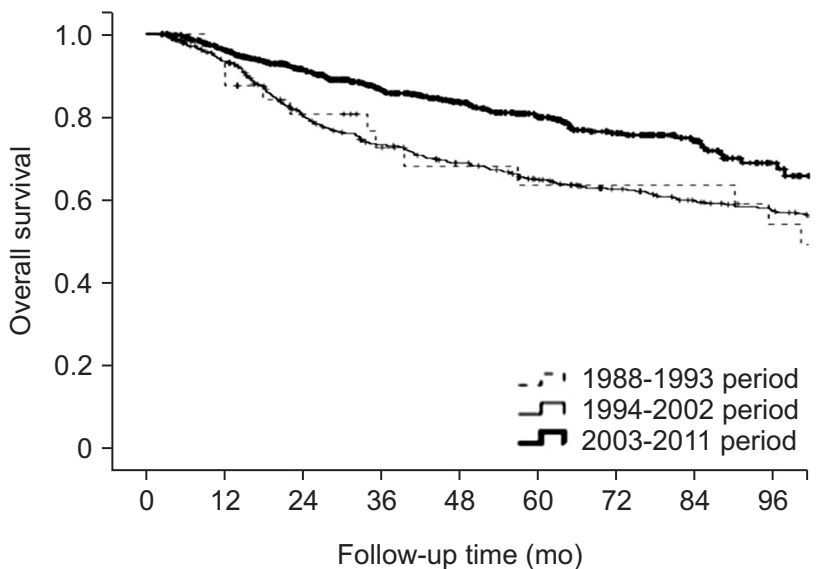

Fig. 2. Overall survival according to period. The 5-year overall survival rates were $63.5 \%$ for $1988-1993,64.8 \%$ for $1994-2003$, and $79.9 \%$ for $2004-2011$ ( $p<0.001)$.

between RT techniques (2DRT, 70.7\%; 3DRT, 88.6\%; and IMRT, $60.3 \%$ ). However, as for dose given to high risk lymphatic chain, $67.4 \%$ of patients treated with 2DRT received below 55 Gy while $64.8 \%$ of patients treated with IMRT received 55-60 Gy. Patients given doses below 55 Gy to low risk lymphatic chain were $70.6 \%$ with 2 DRT and $58.0 \%$ with IMRT. Boost RT was given to 40 patients (2.8\%) using IMRT (1.0\%), stereotactic body radiotherapy $(1.0 \%)$, and brachytherapy $(0.8 \%)$.

\section{Response and treatment outcome}

Responses were evaluated by physical examination (62.1\%), neck CT (56.5\%), neck MRI (32.2\%), PET-CT (14.1\%), and histopathological examination (5.0\%). The number of patients who showed clinical responses was 1,353 (93.6\%), and the more details were as follows: complete response, 1,025 (70.9\%); partial response, $328(22.7 \%)$; stable disease, $35(2.4 \%) ;$ and progressive disease, 15 (1.0\%). The 5 -year OS rates were $73.6 \%$ for the whole group and those for individual stages were as follows: stage I, 96.3\%; stage II, 86.2\%; stage III, 77.4\%; and stage IV, 60.4\%. The 5 -year OS rate showed a significantly better outcome in the third period compared to previous 2 periods (1988-1993, 63.5\%; 1994-2002, 64.8\%; 2003-2011, 79.9\%; $p<0.001$ ) (Fig. 2).

In total, 452 patients (31.2\%) had recurrences: 235 patients (16.2\%) showed locoregional recurrences without distant metastasis, 197 patients (13.6\%) showed distant metastases only, and 20 patients (1.4\%) showed both. Recurrent patients received salvage treatment including surgery $(15.3 \%)$, reirradiation (40.1\%), and chemotherapy (50.0\%). RT techniques used in re-irradiation was 3DRT (46.4\%), fractionated 
stereotactic radiotherapy (23.2\%), or IMRT (21.4\%). Patients received other treatments as follows: 3 patients received CyberKnife-based radiosurgery, 1 patient brachytherapy, 1 patient gamma knife-based radiosurgery, and 1 patient proton therapy.

\section{Discussion and Conclusion}

To the best of our knowledge, this is the first report on the patterns of care for patients with NPC in South Korea. This report is based on the data for a large-scaled multiinstitutional study that were collected from 1,445 patients. We assessed the patterns of care over time and demonstrated that management for NPC patients in South Korea have been improved in accordance with international guidelines. According to the current guidelines, neck $\mathrm{CT}_{\text {, neck MRI, and }}$ PET-CT are recommended for stage evaluation at diagnosis. Although CT provides fundamental anatomic information for defining RT target volume, CT has limitations in exact delineation of tumors that extend to adjacent structures. MRI is superior to CT to detect intracranial involvement, skull bone invasion, or parapharyngeal infiltration. $\mathrm{Ng}$ et al. [6] documented that T-staging was changed in $26.9 \%$ of patients after comparing $\mathrm{CT}$ with MRI and $\mathrm{N}$-staging was upstaged in $6 \%$. Lee et al. [7] also reported that patients staged by MRI before RT achieved significantly higher local tumor control rate and survival rate than the patients staged by CT (MRI staged T3-4 vs. CT staged T3-4: the 5-year local failure-free rate, $83 \%$ vs. $76 \%$; the 5 -year OS, $72 \%$ vs. $63 \%$ ). To better define the anatomical extent, both CT and MRI findings should be incorporated into target contouring. Also, PET-CT has been reported to add value on $\mathrm{CT}$ and $\mathrm{MRI}$ findings, providing metabolic imaging. PET-CT is able to detect metastatic lymph nodes that may appear normal on CT and/or MRI. Kresnik et al. [8] showed that PET led to an upstaging in $20.8 \%$ of patients by detecting lymph node metastases which was negative on CT and MRI. Further, PET-CT provides better sensitivity and specificity of detecting distant metastasis compared to conventional images such as bone scan, abdominal CT or abdominal US $[9,10]$. In our study, MRI and PET-CT had been more frequently used in 2003-2011 compared to previous 2 periods. About three-fourths of patients were evaluated by neck CT, neck MRI and PET-CT in 2003-2011. Performance of the three imaging modality has become the mainstay of diagnostic evaluation.

Changes in treatment regimens over time were also observed. As NPC is not only radiosensitive but chemosensitive tumor, many trials investigated advantage of concurrent chemotherapy for NPC to improve locoregional control. AlSarraf et al. [11] have reported that cisplatin-based CCRT improved the 3-year survival rate from $47 \%$ to $78 \%$ compared to RT alone. After this intergroup study 0099, randomized trials with similar setting confirmed the benefit of CCRT that resulted in better survival outcome than RT alone [12-14]. Update of meta-analysis of chemotherapy in nasopharynx carcinoma (MAC-NPC) reported the results of analysis from 19 trials and 4,806 patients that the addition of concomitant chemotherapy to RT significantly improved OS [15]. Though it was not directly compared in trials or meta-analyses, either weekly cisplatin $\left(30-40 \mathrm{mg} / \mathrm{m}^{2}\right)$ or 3-weekly high-dose cisplatin $\left(100 \mathrm{mg} / \mathrm{m}^{2}\right)$ was thought to be similarly effective. Because only $63 \%$ of patients completed all course of concurrent chemotherapy in intergroup study 0099, effort to set the optimal dose of chemotherapy was done in following studies. Lee et al. [16] reported that dose of cisplatin during CCRT had significant impact on locoregional failure-free rate and OS. The locoregional failure-free rate for patients who received 2-3 concurrent cycles of 3-weekly high-dose cisplatin $\left(100 \mathrm{mg} / \mathrm{m}^{2}\right)$ was significantly higher than those with $0-1$ cycles. Loong et al. [17] showed that patients who received more than 5 cycles of weekly cisplatin $\left(40 \mathrm{mg} / \mathrm{m}^{2}\right)$ had significantly better $0 \mathrm{~S}$ than those who did not. It is generally accepted that a cumulative dose of $200 \mathrm{mg} / \mathrm{m}^{2}$ cisplatin during CCRT is sufficient. In the present study, CCRT was performed on $15.0 \%$ of patients who had stage II-IV disease in 1988-1993; however, it was performed on $78.8 \%$ of patients at the same stages in 2003-2011. For total studied period, the most commonly used chemotherapy regimen for CCRT was cisplatin (81.3\%). Of the 894 patients who treated with CCRT, 749 patients (83.8\%) received more than 5 cycles of weekly chemotherapy or more than 2 cycles of 3-weekly high-dose chemotherapy.

As most NPC patients present with advanced disease at diagnosis and distant metastasis is the main pattern of failure, efficacy of systemic chemotherapy have been studied extensively. Neoadjuvant chemotherapy was tried to reduce tumor burden before RT and to eradicate micro-metastases. Several phase II trials showed high overall response rate of cisplatin-based combination chemotherapy ranging from 75\% to $90 \%$ [18-20]. However, prospective randomized trials failed to demonstrate improvement in survival [21-23]. Adjuvant chemotherapy was included in several trials investigating efficacy of CCRT, because it was performed in intergroup study 0099. Though CCRT followed by adjuvant chemotherapy showed significantly better treatment outcome than RT 
alone, randomized trials evaluating the role of adjuvant chemotherapy demonstrated no survival benefit $[24,25]$. Meta-analysis reported in 2015 by Yan et al. [26], showed no difference for survival outcome between CCRT alone, CCRT after neoadjuvant chemotherapy, and CCRT followed by adjuvant chemotherapy. According to guidelines, CCRT with or without adjuvant chemotherapy is the standard treatment, but neoadjuvant chemotherapy is not recommended [5]. Decision for adjuvant chemotherapy is left for each clinician. Percentages of patients who received neoadjuvant and adjuvant chemotherapy in this study were not much high as $32.4 \%$ and $25.3 \%$, respectively. Both showed a tendency to increase in patients with more advanced stage. Over the period, neoadjuvant chemotherapy declined by almost half, reflecting the negative results of trials. Adjuvant chemotherapy has maintained a similar level after 1994. The most commonly used regimen of adjuvant chemotherapy was 5-fluorouracil/ cisplatin (87.2\%) which was used in intergroup study 0099. The regimens of neoadjuvant chemotherapy were more variable but 5-fluorouracil/cisplatin was the most common (49.8\%). More studies investigating the role of neoadjuvant or adjuvant chemotherapy is needed to propose a clear guideline.

Advances in RT techniques were remarkable over the periods studied. In 1988-1993, 92.5\% of the patients were treated with the 2DRT technique; in 2003-2011, the 3DRT technique and IMRT were performed on $92.0 \%$ of patients. During the whole period of 25 years, the mainstream of RT for NPC patients was changed from the 2DRT through 3DRT, in currently IMRT. RT dose schedules also changed according to advances in RT techniques. Total RT doses given to primary tumor were not quite different between patients treated with 2DRT technique and those treated with IMRT. However, daily doses delivered with 2DRT were 1.8-2.0 Gy in 97.7\% of patients, while with IMRT over $2.0 \mathrm{~Gy}$ in $81.5 \%$. Also, increase of RT doses given to high risk lymphatic chain was observed; $67.4 \%$ of patients received doses below 55 Gy with 2DRT technique but $64.8 \%$ of patients over 55 Gy with IMRT.

In the 2DRT era, doses of 66-70 Gy was delivered to primary tumor via laterally opposed fields. It could deliver tumoricidal doses to the primary tumor, but compromise of target coverage was inevitable because of proximity to critical organs such as brainstem or temporal lobe. Delivery of sufficient dose to lymphatic area was also hardly achievable. The 5 -year local control was reported as 78\%-85\% and the 5-year OS was 50\%-75\% in 2DRT era [7,27-30]. In comparison to 2DRT, IMRT can make highly conformal isodose lines and prevent target volume from being underdosed. This improved dose distributions potentially lead to improved tumor control and better survival outcomes [31,32]. The results of IMRT treated by Lee et al. [33] showed $97 \%$ local progression-free survival and $88 \%$ OS with 4-year follow-up. Owing to the favorable outcomes, IMRT became the major RT technique to treat NPC patients nowadays. The present study revealed that IMRT enables radiation oncologists to elevate daily dose to the gross tumor over $2 \mathrm{~Gy}$, and also elevate total dose given to the high risk lymphatic chain.

This study has the limitation in terms of data quality because missing data were included in the dataset. This was unavoidable because patient data were collected retrospectively through surveys of multiple institutions. However, this study has a large sample size, which sufficiently showed the patterns of care for NPC patients in South Korea. Also, data on RT planning and dosimetry were not collected so the comparison of the RT plans according to RT techniques could not be done.

In conclusion, there have been advances in the management of NPC patients in South Korea. Diagnostic imaging studies including $C T, M R I$ and PET-CT have been more frequently used and CCRT have been performed in more patients. Also, RT techniques and dose prescription have been advanced remarkably to adopt the IMRT technique and dose escalation. Treatment for NPC patients in South Korea reflected the upto-date results of trials and advanced in accordance with the international guidelines.

\section{Conflict of Interest}

No potential conflict of interest relevant to this article was reported.

\section{References}

1. Curado MP, Edwards $B$, Shin HR, et al. Cancer incidence in five continents, vol. IX. Lyon, France: International Agency for Research on Cancer; 2007.

2. Korea Central Cancer Registry, National Cancer Center. Annual report of cancer statistics in Korea in 2012. Goyang, Korea: Ministry of Health and Welfare; 2014.

3. Wilson CP. The approach to the nasopharynx. Proc R Soc Med 1951:44:353-8.

4. Qin DX, Hu YH, Yan JH, et al. Analysis of 1379 patients with nasopharyngeal carcinoma treated by radiation. Cancer 1988 61:1117-24.

5. National Comprehensive Cancer Network. NCCN Clinical Practice Guidelines in Oncology (NCCN Guidelines): head and neck cancers v2.2014 [Internet]. Fort Washington, PA: National 
Comprehensive Cancer Network; c2014 [cited 2014 May 30]. Available from: http://www.nccn.org/professionals/physician_ gls/f_guidelines.asp.

6. Ng SH, Chang TC, Ko SF, et al. Nasopharyngeal carcinoma: MRI and CT assessment. Neuroradiology 1997;39:741-6.

7. Lee AW, Sze WM, Au JS, et al. Treatment results for nasopharyngeal carcinoma in the modern era: the Hong Kong experience. Int J Radiat Oncol Biol Phys 2005;61:1107-16.

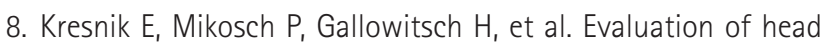
and neck cancer with (18)F-FDG PET: a comparison with conventional methods. Eur J Nucl Med 2001;28:816-21.

9. Chang JT, Chan SC, Yen TC, et al. Nasopharyngeal carcinoma staging by (18)F-fluorodeoxyglucose positron emission tomography. Int J Radiat Oncol Biol Phys 2005;62:501-7.

10. Tang LQ, Chen OY, Fan W, et al. Prospective study of tailoring whole-body dual-modality [18F]fluorodeoxyglucose positron emission tomography/computed tomography with plasma Epstein-Barr virus DNA for detecting distant metastasis in endemic nasopharyngeal carcinoma at initial staging. J Clin Oncol 2013;31:2861-9.

11. Al-Sarraf M, LeBlanc M, Giri PG, et al. Chemoradiotherapy versus radiotherapy in patients with advanced nasopharyngeal cancer: phase III randomized Intergroup study 0099. J Clin Oncol 1998;16:1310-7.

12. Lee AW, Tung SY, Chua DT, et al. Randomized trial of radiotherapy plus concurrent-adjuvant chemotherapy vs radiotherapy alone for regionally advanced nasopharyngeal carcinoma. J Natl Cancer Inst 2010;102:1188-98.

13. Wee J, Tan EH, Tai BC, et al. Randomized trial of radiotherapy versus concurrent chemoradiotherapy followed by adjuvant chemotherapy in patients with American Joint Committee on Cancer/International Union against cancer stage III and IV nasopharyngeal cancer of the endemic variety. J Clin Oncol 2005;23:6730-8.

14. Chen $Y$, Liu MZ, Liang SB, et al. Preliminary results of a prospective randomized trial comparing concurrent chemoradiotherapy plus adjuvant chemotherapy with radiotherapy alone in patients with locoregionally advanced nasopharyngeal carcinoma in endemic regions of china. Int J Radiat Oncol Biol Phys 2008;71:1356-64.

15. Blanchard $P$, Lee $A$, Marguet $S$, et al. Chemotherapy and radiotherapy in nasopharyngeal carcinoma: an update of the MAC-NPC meta-analysis. Lancet Oncol 2015;16:645-55.

16. Lee AW, Tung SY, Ngan RK, et al. Factors contributing to the efficacy of concurrent-adjuvant chemotherapy for locoregionally advanced nasopharyngeal carcinoma: combined analyses of NPC-9901 and NPC-9902 trials. Eur J Cancer 2011;47:656-66.

17. Loong HH, Ma BB, Leung SF, et al. Prognostic significance of the total dose of cisplatin administered during concurrent chemoradiotherapy in patients with locoregionally advanced nasopharyngeal carcinoma. Radiother Oncol 2012;104:300-4.
18. Bachouchi $M$, Cvitkovic $E_{1}$ Azli N, et al. High complete response in advanced nasopharyngeal carcinoma with bleomycin, epirubicin, and cisplatin before radiotherapy. J Natl Cancer Inst 1990;82:616-20.

19. Atichartakarn $V$, Kraiphibul $P$, Clongsusuek $P$, Pochanugool L, Kulapaditharom B, Ratanatharathorn V. Nasopharyngeal carcinoma: result of treatment with cis-diamminedichloroplatinum II, 5 fluorouracil, and radiation therapy. Int J Radiat Oncol Biol Phys 1988;14:461-9.

20. Clark JR, Norris CM Jr, Dreyfuss Al, et al. Nasopharyngeal carcinoma: the Dana-Farber Cancer Institute experience with 24 patients treated with induction chemotherapy and radiotherapy. Ann Otol Rhinol Laryngol 1987;96:608-14.

21. Chan AT, Teo PM, Leung TW, et al. A prospective randomized study of chemotherapy adjunctive to definitive radiotherapy in advanced nasopharyngeal carcinoma. Int J Radiat Oncol Biol Phys 1995;33:569-77.

22. Ma J, Mai HO, Hong MH, et al. Results of a prospective randomized trial comparing neoadjuvant chemotherapy plus radiotherapy with radiotherapy alone in patients with locoregionally advanced nasopharyngeal carcinoma. J Clin Oncol 2001;19:1350-7.

23. Chua DT, Ma J, Sham JS, et al. Long-term survival after cisplatin-based induction chemotherapy and radiotherapy for nasopharyngeal carcinoma: a pooled data analysis of two phase III trials. J Clin Oncol 2005;23:1118-24.

24. Chi KH, Chang YC, Guo WY, et al. A phase III study of adjuvant chemotherapy in advanced nasopharyngeal carcinoma patients. Int J Radiat Oncol Biol Phys 2002;52:1238-44.

25. Chen $L, H u$ CS, Chen $X Z$, et al. Concurrent chemoradiotherapy plus adjuvant chemotherapy versus concurrent chemoradiotherapy alone in patients with locoregionally advanced nasopharyngeal carcinoma: a phase 3 multicentre randomised controlled trial. Lancet Oncol 2012;13:163-71.

26. Yan M, Kumachev A, Siu LL, Chan KK. Chemoradiotherapy regimens for locoregionally advanced nasopharyngeal carcinoma: a Bayesian network meta-analysis. Eur J Cancer 2015;51:1570-9.

27. Carman J, Strojan P. Nasopharyngeal carcinoma in Slovenia, 1990-2003 (results of treatment with conventional twodimensional radiotherapy). Rep Pract Oncol Radiother 2012; 17:71-8.

28. Yeh SA, Tang Y, Lui CC, Huang YJ, Huang EY. Treatment outcomes and late complications of 849 patients with nasopharyngeal carcinoma treated with radiotherapy alone. Int J Radiat Oncol Biol Phys 2005;62:672-9.

29. Yi JL, Gao L, Huang XD, et al. Nasopharyngeal carcinoma treated by radical radiotherapy alone: ten-year experience of a single institution. Int J Radiat Oncol Biol Phys 2006;65:161-8.

30. Lai SZ, Li WF, Chen L, et al. How does intensity-modulated 
radiotherapy versus conventional two-dimensional radiotherapy influence the treatment results in nasopharyngeal carcinoma patients? Int J Radiat Oncol Biol Phys 2011;80:6618.

31. Zeng $L$, Tian $Y M$, Sun XM, et al. Intensity-modulated radiotherapy for stage IVA/IVB nasopharyngeal carcinoma: clinical outcomes and patterns of failure in an endemic area in China. Strahlenther Onkol 2014;190:993-1000.
32. Zong J, Lin S, Lin J, et al. Impact of intensity-modulated radiotherapy on nasopharyngeal carcinoma: validation of the 7th edition AJCC staging system. Oral Oncol 2015;51:254-9.

33. Lee $N$, Xia P, Quivey JM, et al. Intensity-modulated radiotherapy in the treatment of nasopharyngeal carcinoma: an update of the UCSF experience. Int J Radiat Oncol Biol Phys 2002;53:1222. 